\title{
The Design and Application of Engine Vibration Test Platform Based on Virtual Instrument LabVIEW
}

\author{
Teng minjun ${ }^{1, a}$ \\ ${ }^{1}$ Guangzhou College of South China University of Technology, China \\ atengminjun@yahoo.com.cn
}

Keywords: Automotive engine, LabVIEW, vibration test

\begin{abstract}
This paper is based on LabVIEW software to develop a car engine vibration test platform. Completion of the vibration test platform structures, software design of the vibration test platform and test of the vibration test platform, it can analyze radial vibration displacement and power spectra in different speed. It has great practical value for automotive engine damping, and it has certain reference for engine designing and manufacturing.

The engine is the heart of the car. It provides power for the normal operation of the car. It related to the car's power and environmental protection. It is the core components of the engine powered mechanical plant, vehicles, ships and other transportation equipments. It plays a critical role in the normal functioning of the equipment. The type of a car engine in the market is reciprocating piston engine mainly. The engine is prone to vibrate during operation; the reason is reciprocating inertia force and its torque, the rotation inertia force and its torque of crank mechanism, making its output torque unevenness[1,2]. This article is to build a virtual development platform based on LabVIEW, using this platform to get the test data, such as the emissions data, spindle vibration data and engine speed and power data[3].
\end{abstract}

\section{The establishment of the vibration test platform}

The components of the vibration test platform include: automotive engine, couplings, non-contact eddy current displacement sensor, PCI-6221 data acquisition card and PC. The specific test platform is shown in Figure 1.

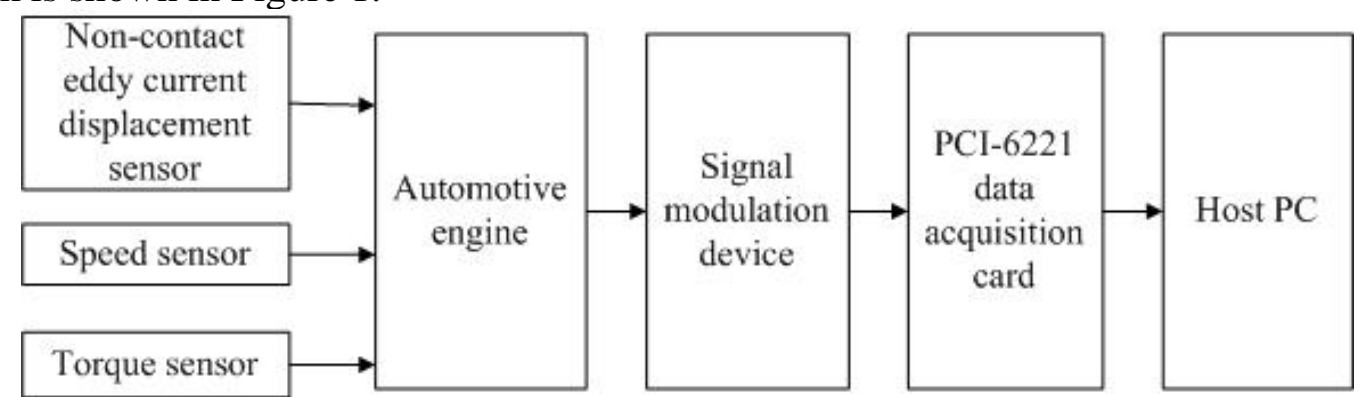

Fig. 1 System diagram of vibration test platform

In this system, three kinds of sensors are used. They are non-contact eddy current displacement sensor, speed sensor and torque sensor. The non-contact eddy current displacement sensor is based on "vortex effect" which is made on the metal surface by high-frequency magnetic field, it is the ideal sensor to detect and control mechanical vibration and displacement. Non-contact eddy current displacement sensor uses the CZF displacement, vibration sensor. For precise data monitoring and controlling, there are 2-way non-contact eddy current displacement sensors installed vertically in the output shaft of the engine to measure the radial vibration. Magnetic-electric engine speed sensor is used to measure the pulse signal of the engine output shaft in order to monitor its speed. Torque sensor is used to measure the torque, converting it into the output power of the automobile engine.

Signal modulation circuit, it makes the analog signal which comes from the sensor amplify, filter and other operations, it can convert the analog signal to the standard signal which the data acquisition card can identify.

NI PCI6221 (37-pin) data acquisition card (DAQ card) has 2-channel 16-bit analog output, its 
maximum sampling rate is $500 \mathrm{kS} / \mathrm{s}$, DAQ card has16-channel analog input with a 37-pin D-Sub. The role of the data acquisition card is $\mathrm{A} / \mathrm{D}$ conversion on the analog signal and then passes to the PC.

PC's function is to receive data transmitted from the data acquisition card, and use the LabVIEW software installed on the PC to display, preserve and analyze the vibration signal, speed signal and power spectral data.

\section{Software designing of vibration test platform}

\section{Program design of vibration test platform}

In the software designing of vibration test platform, we divide the system into various functional modules. In general, software should contain the following modules: 1.signal acquisition module; 2.data read module; 3.data processing module; 4.data storage module; 5.results display module[4]. In order to integrate the individual modules together, we also need to design a main interface to use each module, the overall software design of engine vibration test platform is shown in Figure 2.

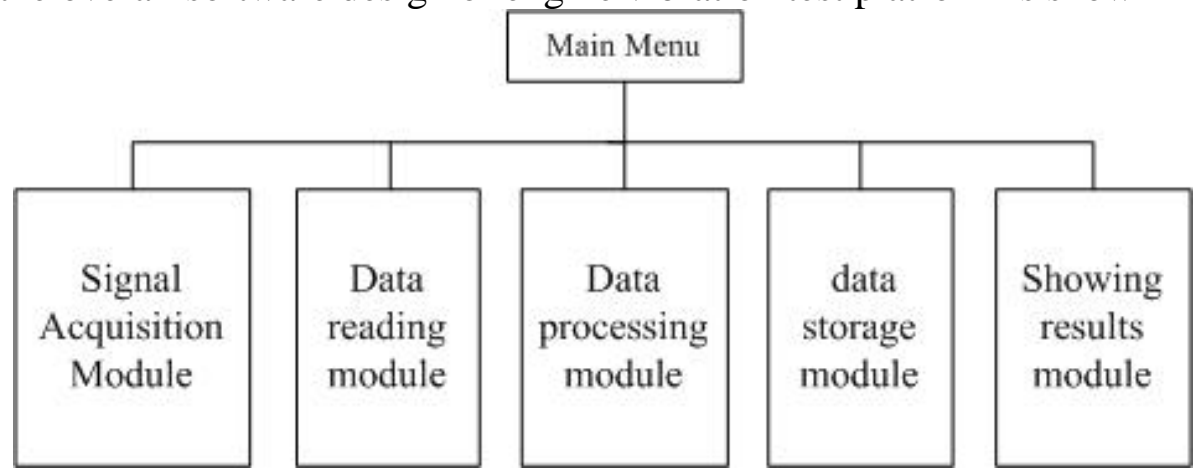

Fig. 2 The overall software design of vibration test platform

\section{The software design of vibration test platform}

The software of vibration test platform is consisted of two parts: the instrument front panel and flow diagram, the front panel is mainly used for data collection, reading, processing, storage and display, the equivalent of the man-machine interface. Flow diagram is the link of each module in testing software, it is a core part of the software, and the software's main interface is shown in Figure 3 and Figure 4 [5].

(1) Acquisition rate module. The module is the collection rate's adjustment of NI PCI6221 (37-pin) data acquisition card (DAQ card), the maximum sampling rate is $500 \mathrm{KHz}$, default value is $250 \mathrm{KHz}$.

(2) Set speed and actual speed module. The module function is to set the rotational speed of the automotive engine, and to load the detected engine speed by the data acquisition card.

(3) Radial vibration displacement module. The module function is to collect the amount of vibration at different speeds with different detection points, provided the raw data for analysis and fitting the following data.

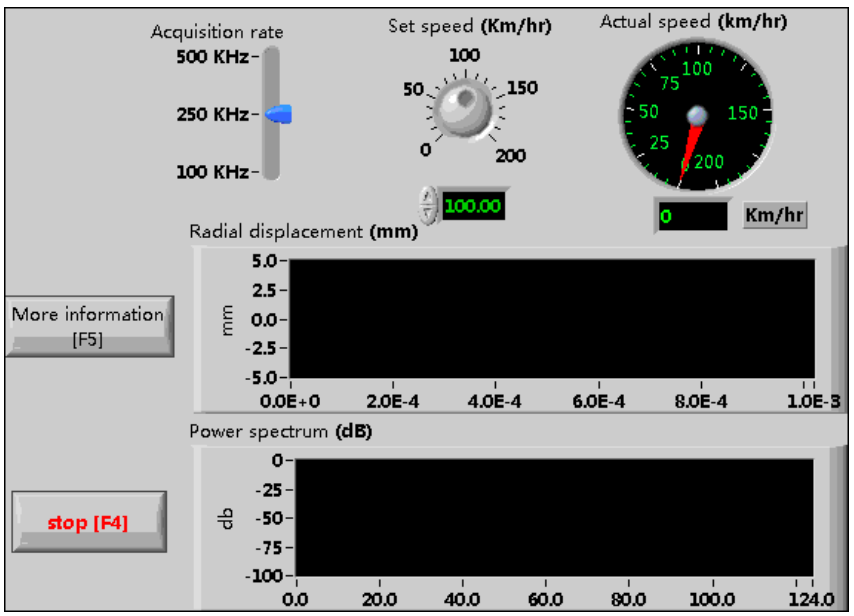


Fig. 3 The front panel of vibration test platform software

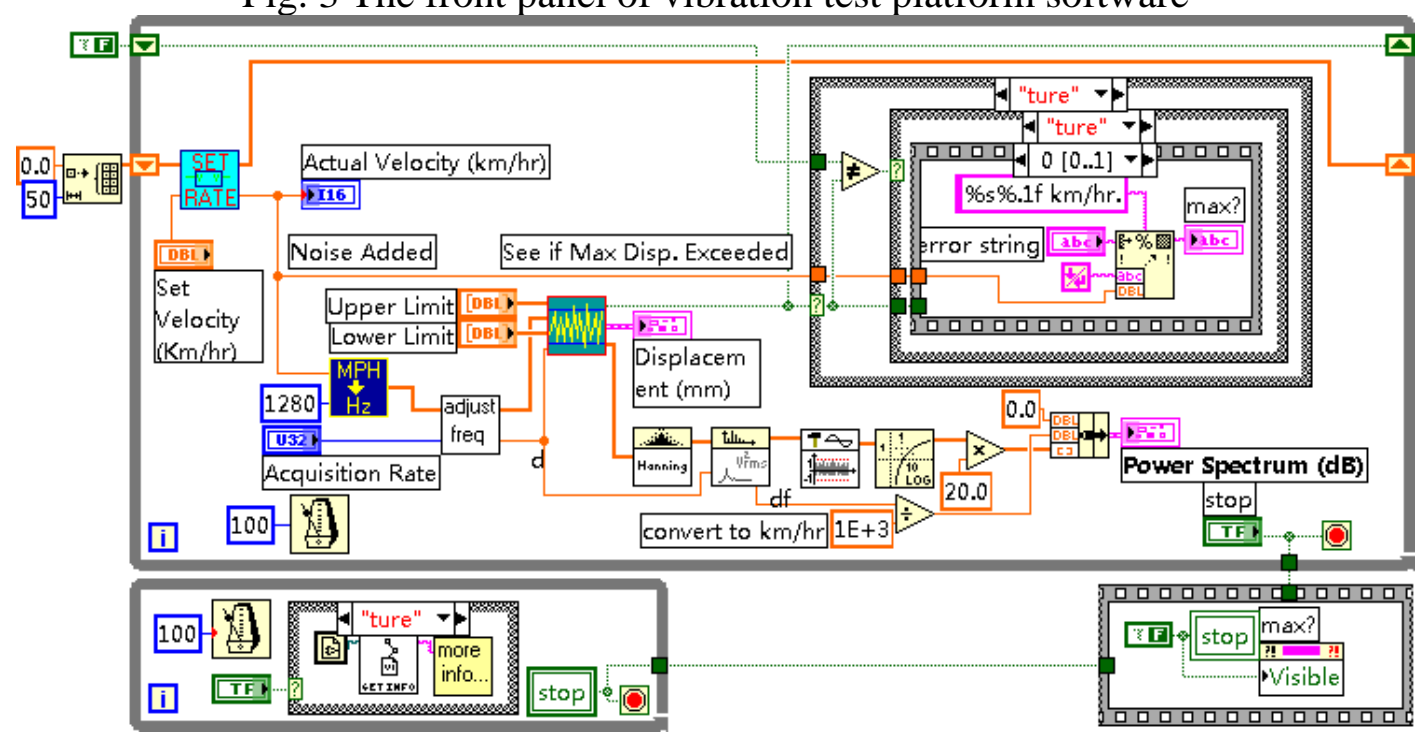

Fig. 4 Software block diagram of the vibration test platform

(4) Power spectrum module. The module function is to use the torque measured by the torque sensor, converts into output power. The formula is shown in Eq.1

$\mathrm{P}=\mathrm{T}^{*} \mathrm{n} / 9550$

$\mathrm{P}$ - power, $\mathrm{KW}$

$\mathrm{n}$ - motor rated speed, $\mathrm{r} / \mathrm{min}$

$\mathrm{T}$ - torque, $\mathrm{Nm}$

\section{Vibration test of vibration test platform}

In order to verify the effectiveness and correctness of the vibration test platform, we use this vibration test platform for certain types of light cars. Test road is a class A road, and it's a dry, clean and flat road. The test is in a straight line sections. Road length is about $2 \mathrm{~km}$, its width is from 8 meters to 10 meters, the road slope is not greater than $0.1 \%$, testing weather condition is no fog, no rain and no wind, relative humidity less than $80 \%$, and the temperature is from 0 to $36^{\circ} \mathrm{C}$.

The vehicle speed are 30, 60 and $100 \mathrm{Km} / \mathrm{hr}$, the sampling frequency is $250 \mathrm{KHz}$, Figure 5 is radial vibration displacement diagram and power spectra diagram in each speed. Test results are the same with the using of measurements about torque, speed and power[6].

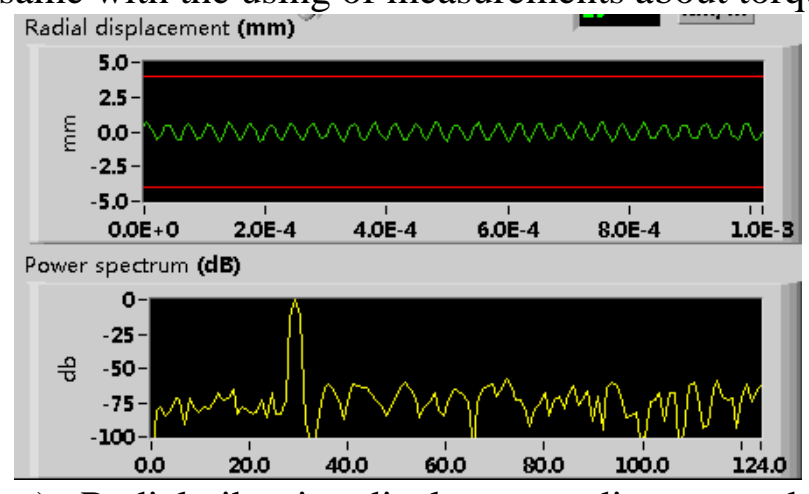

a) Radial vibration displacement diagram and power spectra diagram in $30 \mathrm{Km} / \mathrm{hr}$

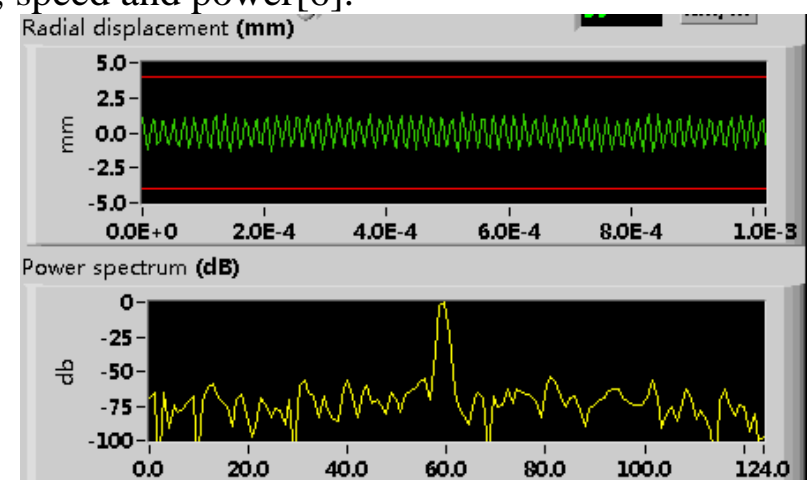

b) Radial vibration displacement diagram and power spectra diagram in $60 \mathrm{Km} / \mathrm{hr}$ 


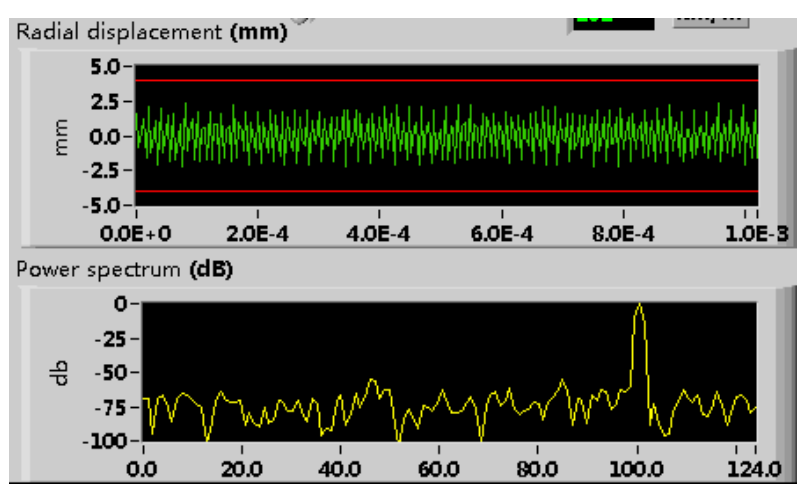

c) Radial vibration displacement diagram and power spectra diagram in $100 \mathrm{Km} / \mathrm{hr}$ Fig. 5 radial vibration displacement diagram and power spectra diagram in each speed

\section{Summary}

(1) Vibration test platform base on LabVIEW can analyze radial vibration displacement and power spectra in different speed, with the development of the PC hardware and software, measurement and control technology base on LabVIEW can be comparable with the traditional monitoring and control equipment, and it has the advantages of low cost and short development cycle.

(2) The test data show that the data collection, statistics and analysis of car engine vibration, can get from vibration test platform based on LabVIEW.

\section{References}

[1] Wang Zhonghai, Lu Ping,Song Enzhe: Diesel digital electronic governor [J]. Diesel, 2006, 28 (2): 24 - 26.

[2] Zhao Yong: virtual instrument software platform technology status and development trend [J] Foreign Electronic Measurement Technology, 2002 (1):2-4.

[3] Li Meilin, Li Yiqiang, Xie Huiming: Car engine vibration test study [J]. Hunan University, 2003, 30 (2): $41-43$.

[4] People's Republic of China national standard GB 7184-87. Small and medium-power diesel engine vibration measurement method.[S]. Beijing: National Bureau of Technical Supervision. 1987. 1-4.

[5] Qi Xingming,Tian Jingjing,XiaNin: LabVIEW Getting Started with the actual development of 100 cases [M] Beijing: Electronic Industry Press, 2011

[6] Chen Min,Tang Xiaoan: Virtual instrument software the LabVIEW and data acquisition [J] small and micro computer systems, 2001, 22 (4): 501 - 503. 